\title{
European approval system for advanced therapies: good news for patients and innovators alike
}
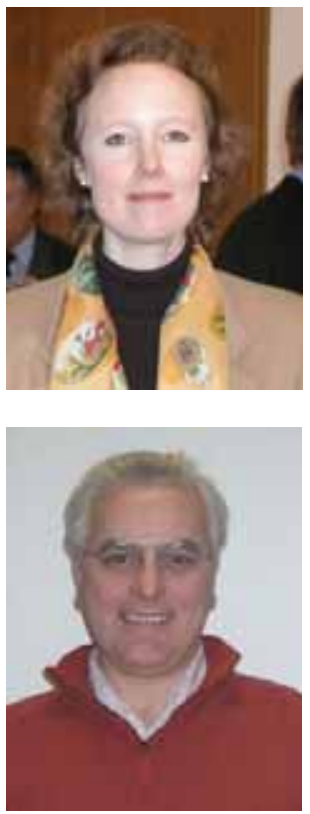

\section{WillsHughes Wilson $^{1} \&$ Duncan Madkay ${ }^{2 t}$}

${ }^{\dagger}$ Author for correspondence ${ }^{1} \mathrm{G}$ enzyme \& Chair, EuropaBio Advanced Therapies Working Group Tel.: +32 27141743 ; Fax: +3227141747

E-mail:willshughes-wilson@ genzyme.com

2D irector Regulatory Affairs Europe Biosurgery, Genzyme Tel.: +44 1223394065 Fax: +44 122339 4191; E-mail: duncan.mackay@ genzyme.com
'The Advanced Therapies Regulation aims to take these products into the wellestablished pha mac eutic al legisla tive system while, at the same time, ensuring that this system is tailored to meet the needs of the products.'

$\mathrm{H}$ armonized regulations in Europe for advanced therapies, comprising somatic cell therapy, gene therapy and tissue-engineered products will, over time, dramatically increase the availability of these innovative new therapies to patients, provide assurance to the general public about the safety of these products, and would also create a stable regulatory climate for innovators.

In N ovember 2005, the European Commission published a legislative proposal for the authorization of 'advanced therapies' at EU level. This new category of products comprises three key groups: somatic cell therapy, gene therapy (both of which are currently regulated under EU pharmaceutical legislation) and tissue-engineered products, a category that is currently unregulated at EU level.

The Advanced Therapies Regulation aims to take these products into the well-established pharmaceutical legislative system while, at the same time, ensuring that this system is tailored to meet the specific nature of the products. It offers manufacturers of these products a single, central evaluation and marketing authorization, simplifying the introduction of products in the entire EU .

Need for a harmonized regulation The regulation of tissue-engineered products has been in discussion for a long time. The lack of regulation at the EU level means that tissue-engineered products are treated in nearly 25 different ways in the 25 (and soon to be 27) EU M ember States. The lack of a comprehensive, clear and uniform regulatory framework creates a highly heterogeneous and segmented market in Europe. 0 wing to the differing requirements, patients may receive products of varying qual ity according to where they live - something that is clearly not in the interest of patient safety. Additionally, the high levels of investment needed to provide appropriate data on these scientifically complicated and high-technology products make it costly to launch products in all M ember States.

Therefore, there have long been calls for a single, EU-level marketing authorization for tissue engineered products, and the European Commission has been working diligently with stakeholders to develop the necessary legislative framework. Initially, proposals had foreseen the creation of a so-called 'third-pillar' approach being neither medical devices nor pharmaceuticals. H owever, when the draft regulation was eventually published in N ovember 2005 , the proposal was to regulate these products under the EU's pharmaceutical framework, for several reasons.

First, many of the elements that were being requested by the stakeholders, such as a single marketing authorization valid throughout the EU, a single evaluation agency, review by a gathered group of the best experts in Europe, clear timelines, support for small companies, are already contained in the pharmaceutical legislation. Second, from a time perspective, the establishment and funding of a new system and a new agency would have taken a lot of time, money and political discussions.

Additionally, by locating tissue-engineered products under the pharmaceutical framework, the draft regulation allows the products and the manufacturers of the products to benefit from many of the support and incentive systems available in the pharmaceutical system. These include, for example, the possibility to apply for 'O rphan' status - under the EU's Orphan Regulation $141 / 2000$, which provides incentives for the research and development of products for rare diseases. They also include a 10 -year period of harmonized regulatory data protection, the possibility to apply for conditional $M$ arketing Authorization and incentives for small \& medium-sized enterprises (SM Es). Furthermore, having products evaluated and approved under the EU's pharmaceuticals framework makes it more likely that these products will be eligible for reimbursement - even if this decision is the competence of the EU M ember States. 
W hile it positions 'advanced therapies' under the pharmaceutical framework, the new regulation proposal does take into account the specific nature of advanced therapies compared with traditional pharmaceuticals. In fact, it foresees a new specialized expert committee in the European Agency for the Evaluation of M edicinal Products (EM EA): the Committee for Advanced Therapies (CAT). This will comprise members from the Committee for M edicinal Products for Human U se (CH M P), as well as experts from a variety of fields. The CAT will be responsiblefor ensuring that the CH M P has access to the necessary specialized expertise to provide scientific recommendation to the European Commission on these products.

\section{Potential shortfalls}

As the proposal goes through the EU 'co-decision' procedure, allowing the European Parliament and the member states to amend it, there are a few areas where stakeholders are hoping for improvements.

First, the proposal foresees a 2-year transition for products that are already on the market to comply with the new provisions. H owever, estimates based on pharmaceutical experience show that it is likely to take as much as 4 years to gather the data and prepare for the application alone.

Second is the issue of hospital-based production of some of these products. Currently, advanced therapies produced by a hospital will be exempted from applying for central EU product $M$ arketing Authorization. This makes sense where a hospital is creating a one off treatment for a specific patient or creating products for research. However, where hospitals act in an industrial manner, producing a product routinely, compliance should be required. The creation of a two-tier system - where products produced by private enterprises are required to undergo rigorous expert scientific evaluation, but where hospitals could do the same with much less evaluation - will pose risks in terms of product reliability and safety. With patient safety in mind, a more common-sense approach would suggest that any advanced therapies produced on a large, routine scale should be required to comply with the new rules.
Clearer definitions of which products are covered by the regulation - specifically so-called 'borderline' products - should be established to help facilitate the development process. Although the CAT has been given the possibility to rule on product classification where there is doubt, this should be the exception rather than the rule.

Europe's SM Es in the advanced therapies sector will need help and support to gain EU approval for their products, as many do not meet all the official EU -wide criteria on SM Es. Therefore, alongside a realistic transition period, enabling them to have the time they need to prepare their application, these companies should also receive increased administrative and financial support. In view of that, the EM EA will need to be sufficiently funded to allow them to offer these incentives.

A key aspect of securing the future of the legislation is to ensure that the regulation focuses exclusively on the quality, safety and efficacy aspects of the treatments under evaluation. D iscussions on ethical considerations should be covered by individual $M$ ember States, since there is no harmonized EU view on ethics. This means all products should be evaluated for safety and effectiveness at theEU level, but individual countries may wish to restrict certain products on their territory on ethical grounds. To attempt to include ethical factors in the product safety evaluation risks further delaying this much-needed regulation.

\section{Conclusion}

The new Advanced Therapies Regulation is an important piece of legislation. It will facilitate the development of a range of new therapies for previously untreatable conditions. It will also support the creation and development of innovative companies in the EU, increase the EU's competitiveness internationally and will ensure that patients can be confident in a universal high level of safety and efficacy from the products they receive. M ost significantly, this regulation will give the highly talented scientific researchers in Europe the opportunity to develop a new range of high-technology, innovative and advanced treatments, bringing solutions for unmet and essential patient needs. 